Review

\title{
Auxin Response Factors Are Ubiquitous in Plant Growth and Development, and Involved in Crosstalk between Plant Hormones: A Review
}

\author{
Xiaohong Kou ${ }^{1,+}$, Xiaoyang Zhao ${ }^{1,+}$, Bingda Wu ${ }^{1}$, Chao Wang ${ }^{1}$, Caie $W u^{2}$, Sen Yang ${ }^{1}$, Jiaqian Zhou ${ }^{1}$ \\ and Zhaohui Xue ${ }^{1, *(D)}$ \\ 1 School of Chemical Engineering and Technology, Tianjin University, Tianjin 300072, China; \\ kouxiaohong@126.com (X.K.); zxy467186123@163.com (X.Z.); ace1716598071@126.com (B.W.); \\ wangchao9582@163.com (C.W.); yangsen950819@163.com (S.Y.); candice0001@163.com (J.Z.) \\ 2 College of Light Industry and Food Engineering, Nanjing Forestry University, Nanjing 210037, China; \\ wucaie@njfu.edu.cn \\ * Correspondence: zhhxue@tju.edu.cn \\ + These authors contributed equally to this work.
}

check for updates

Citation: Kou, X.; Zhao, X.; Wu, B.; Wang, C.; Wu, C.; Yang, S.; Zhou, J.; Xue, Z. Auxin Response Factors Are Ubiquitous in Plant Growth and Development, and Involved in Crosstalk between Plant Hormones: A Review. Appl. Sci. 2022, 12, 1360. https://doi.org/10.3390/ app12031360

Academic Editor: Monica Gallo

Received: 9 December 2021

Accepted: 21 January 2022

Published: 27 January 2022

Publisher's Note: MDPI stays neutral with regard to jurisdictional claims in published maps and institutional affiliations.

Copyright: (c) 2022 by the authors. Licensee MDPI, Basel, Switzerland. This article is an open access article distributed under the terms and conditions of the Creative Commons Attribution (CC BY) license (https:// creativecommons.org/licenses/by/ $4.0 /)$.

\begin{abstract}
Auxin response factors (ARFs) are an important family of transcription factors involved in the exertion of auxin in plants and play a key role in regulating the growth and development of plant nutritional and reproductive organs such as roots, stems, leaves, flowers, fruits, and seeds. Foods of plant origin occupy an important place in the nutritional structure of the human diet, and the main edible parts of different plants vary. In this paper, we review recent research reports on ARFs and summarize its role in the regulation of leaf, flower, root, and fruit growth, as well as other important life activities. We also present the challenges and opportunities that ARFs will present in the future. It will be important to deepen our understanding of the mechanisms by which ARFs interact with other proteins or genes. In addition, it is worth considering that more technical tools should be put into the study of ARFs and that the research should be oriented towards solving practical problems. In the future, it is expected that the nutrition and function of plant-derived foods can be improved through gene editing and other means.
\end{abstract}

Keywords: IAA; auxin; plant hormone; transcription factor; ARF

\section{Introduction}

Auxin is the first discovered plant hormone, one that regulates plant growth and development extensively. The function of auxin depends on a signal transduction pathway involving a variety of transcription factors, one of the most important of which is auxin response factor (ARF). Since the ARF was discovered in Arabidopsis in 1997, ARF has been found in Arabidopsis (Arabidopsis thaliana), rice (Oryza sativa), maize (Zea mays L.), tomato (Solanum lycopersicum), grapes (Vitis vinifera), and other plants. The numbers of ARF genes that have been reported in various species are summarized in Table 1. ARFs were identified as large gene families and showed high homology in the same species. As a key factor in plant auxin signaling, ARFs can specifically bind to the auxin response element (AuxRE) in the promoter region of the auxin response genes and activate or repress the expression of auxin response gene, which in turn triggers many auxin-mediated physiological effects.

Table 1. Summary of the number of ARF genes in species that have been reported.

\begin{tabular}{cc}
\hline Species & Gene No. \\
\hline Agave americana & 32 \\
Ananas comosus & 20 \\
Arabidopsis thaliana & 23 \\
\hline
\end{tabular}


Table 1. Cont.

\begin{tabular}{|c|c|}
\hline Species & Gene No. \\
\hline Aquilegia caerulea & 12 \\
\hline Banana & 47 \\
\hline Beta vulgaris & 16 \\
\hline Boehmeria nivea & 14 \\
\hline Brachypodium distachyon & 24 \\
\hline Brassica rapa & 31 \\
\hline Capsicum annuum & 22 \\
\hline Carica papaya & 15 \\
\hline Cicer arietinum & 28 \\
\hline Citrus clementina & 17 \\
\hline Citrus sinensis & 22 \\
\hline Cucumis melo & 17 \\
\hline Cucumis sativus & 15 \\
\hline Dendrobium officinale & 14 \\
\hline Dimocarpus longan & 17 \\
\hline Eucalyptus grandis & 17 \\
\hline Fagopyrum tataricum & 20 \\
\hline Fragaria vesca & 17 \\
\hline Glycine max & 55 \\
\hline Gossypium raimondii & 35 \\
\hline Hordeum vulgare & 20 \\
\hline Jatropha curcas & 17 \\
\hline Litchi chinensis & 39 \\
\hline Malus domestica & 33 \\
\hline Manihot esculenta & 18 \\
\hline Marchantia polymorpha & 3 \\
\hline Medicago truncatula & 24 \\
\hline Mimulus guttatus & 19 \\
\hline Mulberry & 17 \\
\hline Oryza sativa & 25 \\
\hline Osmanthus fragrans & 50 \\
\hline Phaseolus vulgaris & 25 \\
\hline Phyllostachys edulis & 24 \\
\hline Physcomitrella patens & 12 \\
\hline Populus trichocarpa & 39 \\
\hline Prunus mume & 17 \\
\hline Prunus persica & 17 \\
\hline Prunus sibirica & 14 \\
\hline Rafflesia cantleyi & 9 \\
\hline Ricinus communis & 17 \\
\hline Selaginella moellendorffii & 7 \\
\hline Setaria italica & 23 \\
\hline Solanum lycopersicum & 22 \\
\hline Solanum tuberosum & 20 \\
\hline Sorghum bicolor & 25 \\
\hline Tamarix chinensis & 12 \\
\hline Theobroma cacao & 19 \\
\hline Triticum aestivum & 27 \\
\hline Vitis vinifera & 19 \\
\hline Zea mays & 36 \\
\hline Ziziphus jujuba & 16 \\
\hline
\end{tabular}

ARFs are structurally similar, with most members containing three regions: DBD (DNA-binding domain), MR (middle region), and PB1 (Phox and Bem 1). As shown in Figure 1, DBD is a DNA-binding domain directly involved in binding to AuxRE elements, while the MR region is responsible for activation or repression of target genes. It is believed that the amino acid composition and sequence length of MR region determine whether activation or inhibition. If this region is rich in proline, serine, and threonine it is able to 
produce inhibition, while activator-class ARFs often have glutamine-rich and leucine-rich MR regions. The PB1 domain can bind to Aux/IAA proteins to form heterodimers [1,2]. Some reports show that Aux/IAA and ARFs can form not only dimers but also larger complexes (oligomers), noting that oligomerization of Aux/IAA proteins may be essential for the inhibition of ARF proteins and only sufficient amounts of Aux/IAA proteins can exert the inhibitory effect of ARF proteins. The PB1 domain contributes to the oligomerization of ARFs and is important for Aux/IAA-ARF-mediated gene regulation [3,4]. The mode of action of ARF in auxin signaling is shown in Figure 1: When the cellular auxin concentration is low, Aux/IAA, encoded by the auxin early response gene family, binds to ARF and inhibits the activity of ARFs. When the auxin concentration is increased to a certain level, ubiquitinated Aux/IAA proteins are rapidly hydrolyzed. Aux/IAA protein's inhibition of ARF transcription factors is relieved, and ARFs then promote or repress the expression of downstream genes.
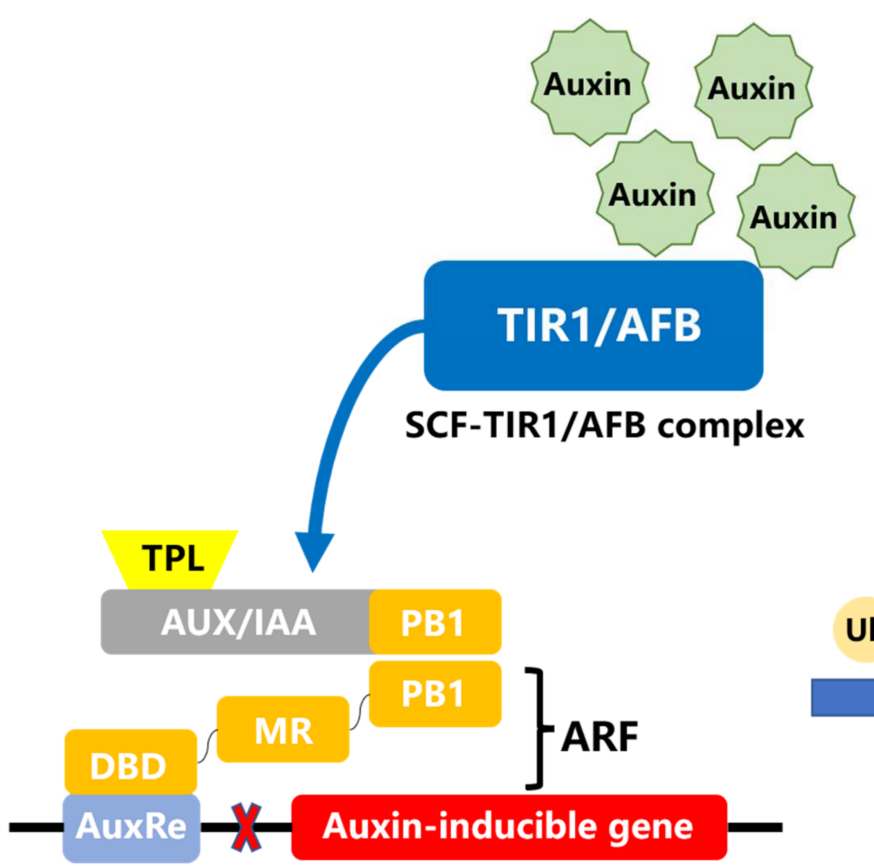

Transcription repressed

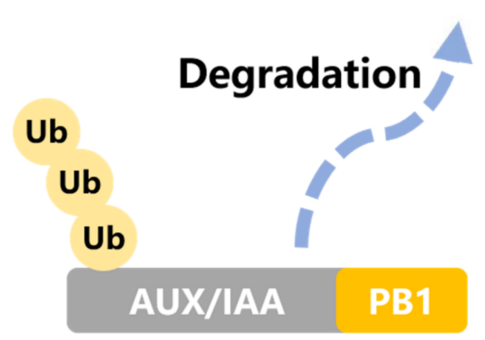

Ub
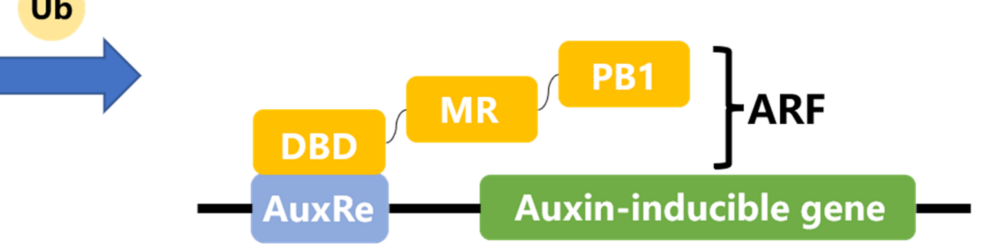

Transcription active

Figure 1. Model of ARF involvement in auxin response. The process of auxin signaling is coordinated by the auxin receptor TIR1/AFB, as well as the signal response factors AUX/IAA and ARF, which together mediate the auxin signaling response. Auxin acts as a "molecular glue" and facilitates the interaction between SCF-TIR1/AFB and Aux/IAA proteins. At low concentrations of auxin, the AUX/IAA repressor binds to the ARF transcription factor and forms a dimer that recruits the co-repressor TPL (TOPLESS) to inhibit the ARF activity and the expression of auxin responsive genes. When the concentration of auxin is increased, Aux/IAA binds to the SCF TIR1/AFB complex and is ubiquitinated and then degraded by $26 \mathrm{~S}$ protease, and then ARF transcription factors are released to activate the transcription of downstream genes. DBD, DNA-binding domain; MR, middle region; PB1, Phox and Bem 1.

ARF is commonly expressed in all periods of plant growth and development and in different parts of the plant, which also determines its comprehensive influence on plant life activities. In addition, Estrada-Johnson et al. [5] found the gene family of strawberry (Fragaria ananassa) auxin response factors (FaARFs) were differentially expressed in the receptacle at four developmental stages by RNAseq. A large number of studies have shown that ARF is involved in the regulation of a variety of plant life activities, including leaf, flower, root development, and fruit ripening, senescence, and shedding. ARF is also involved in stress response and anthocyanin metabolism in plants. In addition, ARFs are 
also involved in plant hormone crosstalk, and the relevant research results are valuable for us to fully understand the regulatory effects of plant hormones. In recent years, studies on the regulation of plant life through hormone signaling pathways have been carried out, and increasingly more genome-wide identification and expression analyses of ARF gene families in plants have been reported, as shown in Table 1, which are of great significance for a more comprehensive understanding of the functions of ARF. In this paper, we summarize recent research reports on the regulatory role of ARF in plant growth, discuss the key roles of ARF in the development of a variety of important organs in plants, propose challenges and opportunities for current ARF-related research, provide inspiration and references for future ARF research, and lay the foundation for the establishment of a functional network of auxin response factors.

\section{ARF Is Involved in Regulating Leaf Development}

Most plants can perform photosynthesis by themselves, absorb carbon dioxide and release oxygen at the same time, and synthesize organic substances. This process is the prerequisite for all organisms on the earth to obtain energy. Leaves are the most important place for photosynthesis of plants. Important indicators such as the shape, size, and chlorophyll content of plant leaves usually determine whether photosynthesis can proceed normally and play a key role in the efficiency of photosynthesis [6]. In addition, in the leaves of plants, there are usually many other physiological activities, such as respiration. Generally speaking, there are stomata on the back of the leaves to support high-intensity respiration. Plants mainly maintain their own metabolic balance through these two activities on the leaves to maintain vitality. In addition, the transpiration also requires the participation of leaves.

At present, there have been a large number of reports showing the plant auxin plays a critical role in the life activity of leaves. A moderate increase in the concentration of auxin will stimulate the growth of leaves, while exceeding the optimal concentration will have an inhibitory effect. Auxin response factor (ARF), as a key regulator in the process of auxin signal transduction, directly participates in the regulation process of auxin on plant leaves in many links $[7,8]$. Arabidopsis thaliana is one of the first species to be used to study the regulation of ARF. In Arabidopsis, AtARF is already a well-studied member of the ARF family, and its regulatory role in leaves has been widely reported. AtARF2, AtARF3, AtARF4, and MONOPTEROS (MP)/ARF5 are involved in the regulation of auxin-responsive gene expression and leaf development. MP can promote the expression of WUSCHEL-RELATED HOMEOBOX1 (WOX1) and PRESSED FLOWER (PRS) directly, promoting leaf flattening. However, the expression of WOX1 and PRS can be repressed by several other members of the ARF family, AtARF2/3/4, as shown in Figure 2. These ARFs are all able to bind directly to the promoters of WOX1 and PRS [6]. Schuetz et al. [7] reported that ARF7 and ARF3 act synergistically with ARF5 in leaf formation, indicating a synergistic interaction, and these gene combinations work in parallel to maintain meristem organization and induce leaf formation. In addition, some studies have indicated that auxin induces leaf expansion by activating NPH4/ARF7 and ARF19 [8] (Figure 2). In tomato, Wu et al. [9] found that tomato leaf development was co-regulated by several members of the ARF family, including SIARF6A/8A/8B/24. In addition, SlARF6A/24 can influence the process of leaflet initiation by binding to the promoter of PIN-FORMED1 (SIPIN1), which affects the local accumulation of auxin. There are also reports that the chlorophyll content in leaves and fruit is significantly increased in tomato mutant lines of overexpressing SIARF6A. While downregulation of SIARF6A reduces the chlorophyll content [10]. ARF also plays a significant role in the leaves of some other species. In rice, OsARF19 controls rice leaf angles by binding to the promoters of the auxin early response gene OsGH3-5 and brassinosteroid (BR) response gene OsBRI1 [11]. 


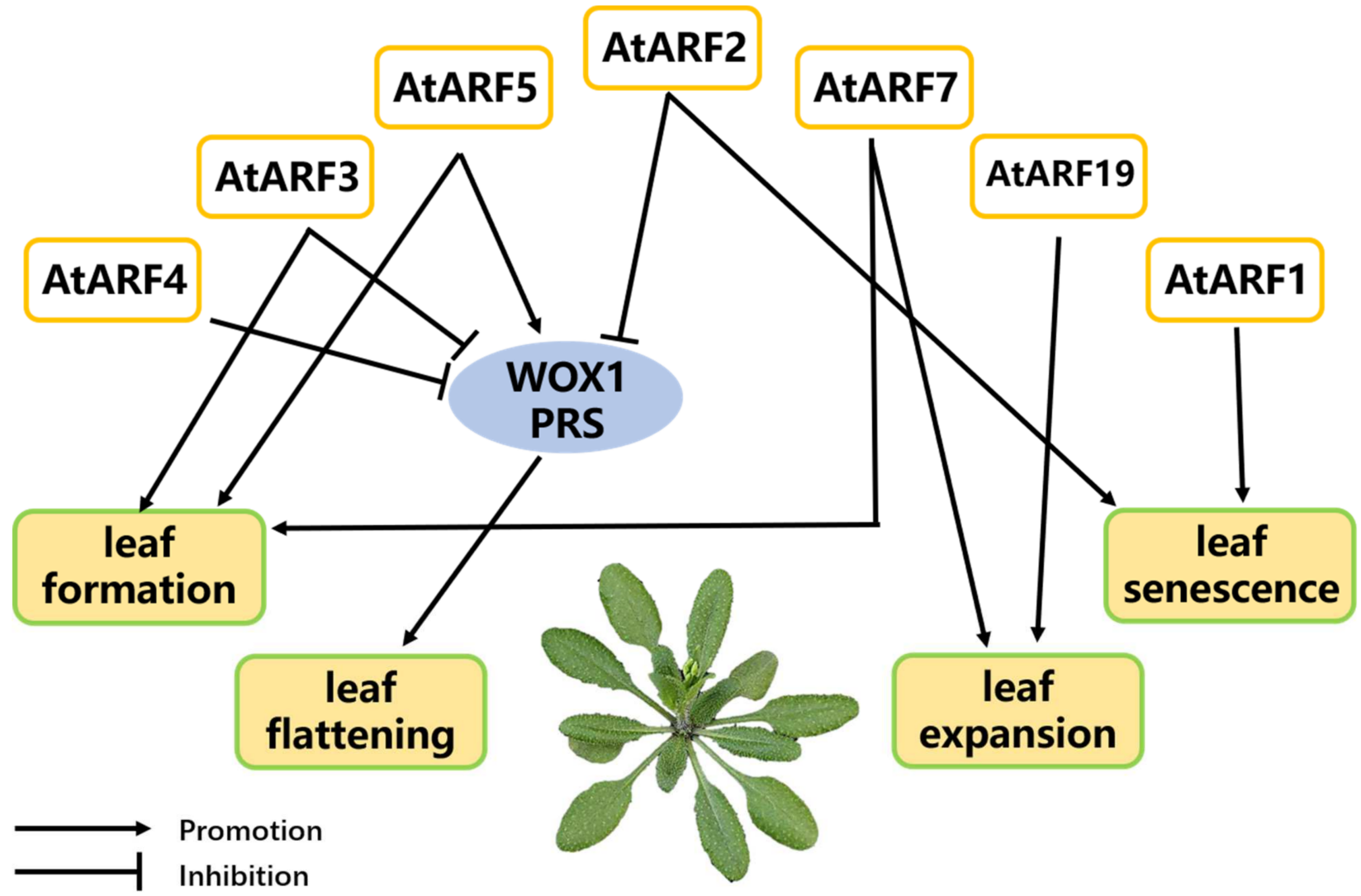

Figure 2. A model of ARFs involved in leaf development and senescence in Arabidopsis thaliana. $\mathrm{ARF} 2 / 3 / 4 / 5$ regulates leaf flattening by targeting the promoters of WUSCHEL-RELATED HOMEOBOX1 (WOX1) and PRESSED FLOWER (PRS). ARF3/5/7 synergistically regulate the leaf formation process. ARF 7 and 19 affect leaf expansion. ARF1 and 2 promote leaf senescence.

Leaf senescence is a necessary process of plant metabolism, accompanied by physiological changes such as the decrease of ribonucleic acid content, the destruction of chloroplasts, and the decrease of photosynthesis capacity. ARF also plays a regulatory role in the process. The AtARF1 and AtARF2 genes in Arabidopsis are involved in regulating leaf senescence, which is independent of the ethylene and cytokinin pathways [12,13]. In tomato, overexpression of SlARF2 accelerates leaf senescence [14]. In litchi, Zhang et al. [15] concluded that LcARF2 may be related to the senescence of litchi leaves.

\section{ARF Is Involved in Regulating Root Development}

Roots are the nutritional organs of plants, usually located below the ground surface, and are responsible for absorbing water and dissolving inorganic salts from the soil, as well as supporting, reproducing, and storing and synthesizing organic matter. Wang et al. [16] reported that microRNA160-targeted AtARF10/AtARF16 plays a crucial role in root cap formation. In rice, OsARF1 is also able to regulate crown roots [17]. Another ARF of rice species is OsARF12, which plays an active role in promoting root elongation [18].

The formation of lateral roots plays a key role in plant development, as well as the ability of plants to obtain nutrients depends on the degree of root branching. Numerous studies have suggested that auxin is a common signal for the formation of lateral roots. In Arabidopsis, AtARF5 is essential for lateral root formation [19]. In the root tissue of seedlings, AtARF7 and AtARF19 are transcriptional activators of early auxin response genes and control the expression of most plant auxin response genes. They regulate lateral root formation through direct activation of LBD/ASLs, as well as AtARF7 and AtARF19 double mutations caused impaired hypocotyl and root development [20]. In tomato, SlARF2 
regulates the formation of lateral roots, and high expression of SIARF2 promotes lateral root growth [14].

In addition to lateral roots, ARF genes also play an important role in adventitious root development. AtARF6 and AtARF8 genes regulate adventitious root formation with the involvement of micro160 and micro167, and AtARF6 has been shown to positively control the development of adventitious roots. It has also been shown that AtARF17 is involved in this process and negatively regulates adventitious roots in Arabidopsis [21]. In rice, crown rootless1 (OsCRL1) encodes a plant-specific protein, and OsARF1 binds directly to AuxRE in the OsCRL1 promoter, which plays an important role in adventitious root initiation [22]. In addition, Yang et al. [23] reported PeARFs may play diverse regulatory roles in adventitious root development of Populus.

The functions of auxin in primary root formation have also been studied for a long time in the field of developmental biology. In Arabidopsis thaliana, primary root formation in early embryogenesis of is initiated with the specification of a single cell called hypophysis. This initial step requires the auxin-dependent release of the transcription factor MONOPTEROS (MP)/ARF5 from its inhibition by the Aux/IAA protein BODENLOS (BDL)/IAA12 [24]. In addition, Dastidar et al. [25] found that MP may play a role by directly regulating the expression of miR390 in Arabidopsis thaliana primary root meristem. Schlereth et al. [26] showed that MP controls embryonic root initiation by regulating a mobile transcription factor.

\section{ARF Is Involved in the Regulation of Floral Structure and Sexual Reproduction}

A plant's flowers are essential for its own reproduction and are the organs of sexual reproduction for the vegetation. Over the course of evolution, the flower of a plant has developed into a more stable structure, generally consisting of a stalk, calyx, receptacle, corolla, pistil, and stamens. The stamens and pistils are composed of different parts, with the stamens consisting of the anthers and filaments and the pistils consisting of the style, stigma, and ovary. Flower development, maintenance, abscission process, and the normal formation of pistil and stamen are all crucial for plant growth and fruit formation. It has been extensively reported that auxin response factors play a key regulatory role in these processes.

Research on the regulatory role of ARF in plant flowers has been carried out mostly in Arabidopsis. Ellis et al. [12] reported that AtARF1 and AtARF2 in Arabidopsis affect flower initiation, stamen development, and flower senescence and abscission. arf2 mutant plants show delays in flowering and abscission of floral organs. arf1 mutations enhanced many arf2 phenotypes, indicating that ARF1 acts in a partially redundant manner with ARF2. AtARF3 regulates floral meristem determinacy by repressing cytokinin biosynthesis and signaling, and loss of AtARF3 leads to the development of pistil appearance defects [27]. There are many reports about the crucial roles of MONOPTEROS (MP)/ARF5 in the regulation of flower formation and other activities of shoot apical meristem. For example, Yamaguchi et al. [28] uncovered a molecular framework for flower initiation that includes AtARF5 and LEAFY, a master regulator of flower development, which reveals a mechanistic link between flower primordium initiation and subsequent steps in flower morphogenesis. AtARF6 and AtARF8 are thought to control stamen and pistil maturation, respectively, and the loss of a single gene in either ARF6 or ARF8 causes delayed stamen filament elongation and delayed anther dehiscence, resulting in reduced self-pollination. The double mutant, on the other hand, is directly and completely sterile [29]. Another study on AtARF8 stated a simple variant of ARF8 can regulate the elongation of stamens [30]. Uniquely, AtARF17 is essential for pollen wall patterning in Arabidopsis by modulating primexine formation at least partially through direct regulation of CALLOSE SYNTHASE5 (CalS5) gene expression [31].

ARF regulation of floral structure and sexual reproduction has been reported in other species. In tomato, overexpression of SIARF2 leads to floral organ senescence [32]. Another study claimed that SIARF6 and SIARF8 downregulated by MicroRNA167 may lead to 
abnormal flower development and female sterility in tomato [14]. In addition to this, Kumar et al. [33] found that SIARF1, SlARF9, SlARF11, SlARF15, and SlARF16 were highly expressed during flowering, suggesting that these genes may be associated with flower development in tomato. Sun et al. [34] found that GrARF9 in cotton influences reproductive stages through its involvement in flower development and postulated a function consistent with that of SlARF9 in tomato. Both FvARF6 and FvARF8 are highly expressed early in strawberry fruit formation, and they may be important for flowering and other reproduction of strawberries [13].

\section{ARF Is Involved in Regulating Fruit Development and Ripening}

Research on fruit development and ripening has always been a hot topic. Human demand for high-quality fruits drives researchers to conduct in-depth research on the formation, development, and quality improvement of fruits, and its economic value is also huge. Plant fruits generally include two parts: pericarp and seed. Pericarp can be divided into exocarp, mesocarp, and endocarp, and seeds play a key role in the process of propagation and reproduction. The process of fruit development depends on cell division and cell expansion. After the completion of the pollination and ovary fertilization process, the carpel tissue around the ovary begins to expand rapidly [35]. The research on fruit includes the entire process from the occurrence of flowers to the later fruit preservation. For a long time, it has been believed that plant hormones play an important regulatory role in fruit growth and development, especially auxin, ethylene, and abscisic acid.

Some progress has been made in recent years regarding the role of ARF in the regulation of tomato fruit development and ripening. Figure 3 shows a model of ARFs regulating tomato fruit development and ripening. SIARF2 is an important part of tomato maturation regulatory network. Overexpression of SIARF2 enhances the expression of ethylene biosynthesis genes, increasing ethylene production and accelerating fruit ripening. However, downregulation or silencing of SIARF2 will lead to maturation defects and even inhibit maturity [36,37]. SIARF4 affects many physiological indicators of tomato fruits. For example, GOLDEN2-LIKE (GLK) transcription factors are required for the regulation of chloroplast and chlorophyll levels [38], and SIARF4 may control the accumulation of chlorophyll and the structure of the fruit cell wall through transcriptional inhibition of GLK1 expression in tomato fruits, as shown in Figure 3. Chlorophyll content is a key feature of immature fruit that affects the nutrient composition and flavor of mature fruit. Inhibition of SlARF4 expression can make tomato fruits show a dark green immature phenotype, and the resulting fruit is harder than the wild type. In addition, SIARF4 is also a major participant in mediating the control of auxin on sugar metabolism [39]. SIARF10 plays a similar role to SIARF4 and regulates chlorophyll and sugar accumulation during tomato fruit development through transcriptional activation of SIGLK1 [40]. SlARF10 is also involved in seed development and the regulation of fruit size and shape [41]. Another auxin response factor that has an effect on the chlorophyll content of tomato fruits is SIARF6A. The chlorophyll content in fruit was significantly increased in tomato mutant with overexpression of SlARF6A. In addition, ethylene production was inhibited, and fruit ripening rate decreased [10]. When pollination occurs, SlARF5 plays a role in fruit germination [42]. SIARF7 negatively regulates both fruit set and fruit development, slowing the plant auxin response during fruit growth $[43,44]$. In addition, SlARF8 and SlARF9 in tomato play a role in regulating fruit development and growth, and in negatively regulating cell division during early fruit development, respectively $[40,45]$. 


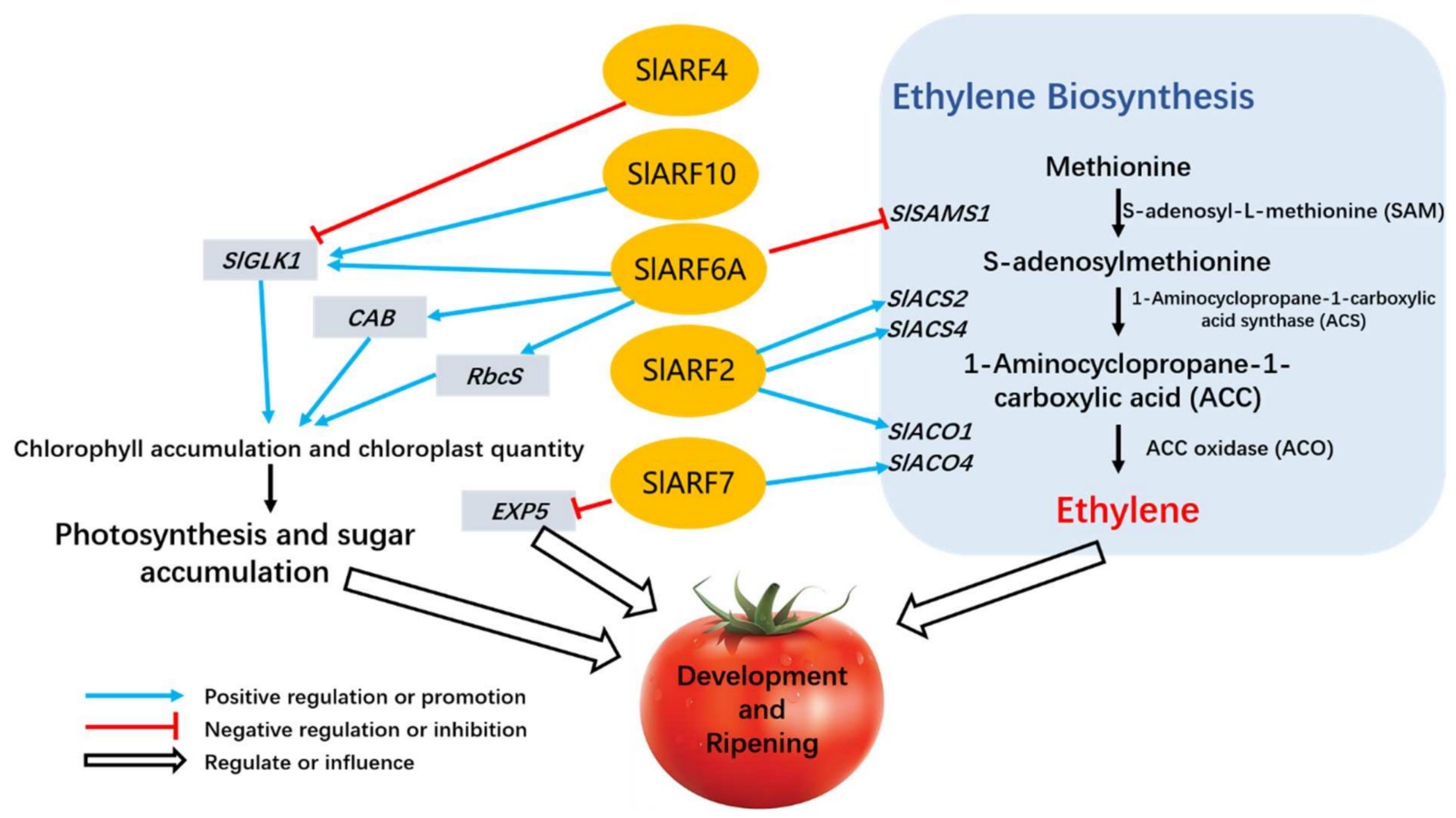

Figure 3. A model of ARFs regulating tomato fruit development and ripening. Several members of the auxin responsive factor family influenced the development and ripening of tomato fruits by regulating the expression of genes related to ethylene biosynthesis and chlorophyll accumulation. SlARF2 regulates ethylene synthesis and tomato ripening by regulating the expression of ethylene biosynthesis genes ACO1/ACS2/ACS4. GOLDEN2-LIKE transcription factor SIGLK1 plays an important role in chloroplast formation and chlorophyll accumulation. SlARF4 inhibits the expression of its encoding gene SIGLK1, while SlARF10 and SlARF6A promotes its expression. Moreover, SlARF6A also targets the promoters of CAB (genes encoding chlorophyll A/B binding) and RbcS (gene encoding ribulose bisphosphate carboxylase small chain), genes that affect chlorophyll accumulation, and positively regulates the expression of these genes. SIARF6A is also a negative regulator of SAMS1, which in turn negatively regulates ripening and ethylene biosynthesis in tomato fruit. EXPANSIN5 (EXP5) likely promotes cell expansion during fruit development. SIARF7 inhibits tomato fruit set and early development by directly regulating EXP5 and ACO4.

A number of auxin response factors have also been reported in other species, and they also impact fruit development and ripening. In Arabidopsis, AtARF2 regulates silique ripening, whereas AtARF8 is a negative regulator of fruit development [12,46]. In strawberries, FaARF6a is considered the most likely candidate among ARFs involved in the regulation of auxin activity in mature flower receptacles, interacting with FaAux/IAA11 [5]. Similar to SIARF4 in tomato, PpARF4 in peaches have an effect on sugar metabolism and cell wall degradation, and thus may be involved in regulating fruit ripening. According to Li, CiARF1 and CiARF18 in citrus sinensis are present in the peel and pulp, respectively, and have constitutive expression patterns during fruit development and ripening [47]. At present, there are few studies on the regulation of auxin in fruit shedding, and some economic fruit trees, such as litchi, are suffering from fruit shedding in actual production. Studies conducted by Zhang showed that the ARF family genes in litchi might play opposite roles in the regulation of litchi fruit abscission, with the majority of the members acting as repressors. The members that repress fruit abscission include ARF2D/2E, 7A/7B, 9A/9B, and 16A/16B, while members such as LCARF5A/B may promote fruit abscission in litchi [15]. It has also been reported that LCARF1 is negatively correlated with small fruit abscission [48]. These findings fill a gap in the research on ARFs regulation of litchi fruit abscission and provide new insights into the function of ARFs. 
It is well known that anthocyanins have antioxidant and free radical scavenging functions and are increasingly sought after by people for their role in reducing fatigue, anti-aging, and beautifying the skin. Studies have shown that ARFs are involved in the regulation of anthocyanin biosynthesis in apples, and MDARF2 and MDARF13 are considered to be inhibitors involved in anthocyanin biosynthesis and metabolism [49,50]. However, similar regulatory effects of auxin in fruits with high anthocyanin content, such as mulberry, blueberry, and blackcurrant, have been rarely reported. It will be very valuable to promote related work in the above-mentioned fruits.

\section{ARF Is Involved in Regulating Stress Response}

Abiotic stresses are the adverse effects of abiotic factors on plants, such as drought, flooding, salinity, mineral deficiencies, and excessively high or low ambient temperatures. Abiotic stresses affect plants in a variety of ways and can affect plant viability and development, which may result in changes in plant growth and crop yield, as well as disturbance of physiological processes such as photosynthetic rates or mineral uptake rates. Throughout their long evolutionary history, plants are constantly improving their ability to combat the adverse factors in the environment. The effects of stresses on plants, especially the negative ones, have indirectly affected human access to food and safety of life, and thus the study of various stresses in plants is also a hotspot.

Numerous studies have shown that auxin response factor ARF plays an important regulatory role in abiotic stress responses in a variety of plants. In Arabidopsis, AtARF7 and AtARF19, members of 23 AtARFs, were suggested to be involved in the regulation of membrane lipid remodeling under phosphorus starvation stress, enhancing the low phosphorus adaptation in Arabidopsis [51]. SlARF4 is involved in the regulation of the response of tomato to salt and osmotic stress by decreasing stomatal conductance as well as increasing relative water content and abscisic acid content of leaves, and thus that tomato exhibits a higher tolerance to salt and osmotic stress [52]. Some other members of SIARFs, such as SIARF1, SIARF4, S1ARF6A, S1ARF7A, S1ARF8A, S1ARF9A, S1ARF18, and S1ARF19, can respond to abiotic stresses at the transcriptional level [53]. Rice is an important food crop worldwide, and OsARFs play a crucial role in the response of rice to abiotic stress. Both OsARF11 and OsARF15 exhibited unique expression patterns under salt stress, indicating that their involvement in rice response to salt stress [54]. Du et al. [55] concluded that most OsARF genes were inhibited by both cold and heat stress, with OsARF4/14/18/19 induced by cold stress and OsARF11/13/16 induced by heat stress. Nine ARFs genes were found to be involved in the response to drought stress, namely, OsARF2/4/10/14/16/18/19/22/23 [53]. In other plants, Chen et al. [56] reported that NtARF6 and NtARF8 mediate plant growth and response to phosphorus starvation stress by regulating reactive oxygen species (ROS) steady-state and phosphorus uptake in tobacco. In Ipomoea batatas, IbARF5 is regulating salt tolerance and drought tolerance in plants [57].

Biotic stresses are imposed by other organisms, such as pests, diseases, and weeds. Compared to the involvement of ARF in plant abiotic stress responses, less research has been conducted on the role of ARF in biotic stress responses. In studies of plant responses to biotic stress, it is relevant to report that in tomato biotic stress the SlARF3 gene's expression level is upregulated [33]. In rice, OsARF17 may be involved in plant defense responses to different types of arboviruses [11]. In fact, the effects of biotic stress are more serious, often resulting in crop yield, loss of fruit nutritional value, and even harm to human health. Therefore, study of ARF's participation in plant biotic stress response will be a valuable research direction in the future, potentially providing us with new solutions to practical production problems.

\section{ARF Is Involved in Crosstalk between Plant Hormones}

ARFs are involved in regulating many physiological processes in plant by mediating auxin signaling. However, ARFs can function in more ways than this. Many studies have 
been carried out on the involvement of ARFs in the crosstalk between plant hormones, providing new insights and ideas for the function of ARFs.

The regulation of plant growth and development by plant hormones is complex, and more than one plant hormone is often required to act simultaneously. The crosstalk and synergy of plant hormones have high research value. In recent years, some results have been obtained on the involvement of auxin response factors in phytohormone crosstalk. In terms of synergistic effects of plant hormones, ATARF6/8, ATARF2/19, and ATARF2 are considered to be key factors linking auxin with jasmonic acid, ethylene, and abscisic acid [58,59]. Liu et al. [60] proposed a model for the effect of ABA-auxin interaction on seed dormancy/germination control; as shown in Figure 4, the strength of the auxin signal determines whether the response factor ARF10/16 can be released from inhibition, which in turn initiates the regulatory effect on the key factor ABSCISIC ACID INSENSITIVE 3 (ABI3) in the downstream ABA pathway, indirectly regulating seed dormancy or germination. In tomato, SIARF5/7 and SIARF2/6A were reported to be associated with auxin synergism with gibberellic acid (GA) and ethylene [10,36,42]. In the previous sections, we mentioned that SIARF7 has an inhibitory effect on tomato fruit set and development, and this effect is achieved precisely through the signaling crosstalk between auxin and gibberellin and ethylene. Hu et al. [44] found that auxin signaling repressor complex SIARF7/SIIAA9 and GA signaling repressor SIDELLA additively inhibit tomato fruit set and fruit development by repressing the expression of growth-related genes and activating ethylene biosynthesis gene (ACO4). In apple, auxin induces ethylene biosynthesis in apple fruit by activating the expression of MdARF5. MdARF5 directly binds to the promoters of ethylene biosynthesis genes $M d A C S 3 a, M d A C S 1$, and $M d A C O 1$ to induce their expression [61]. Moreover, overexpression of IbARF5 upregulates ABA biosynthesis genes in Ipomoea batatas [57].

\section{Auxin signaling inactivation}

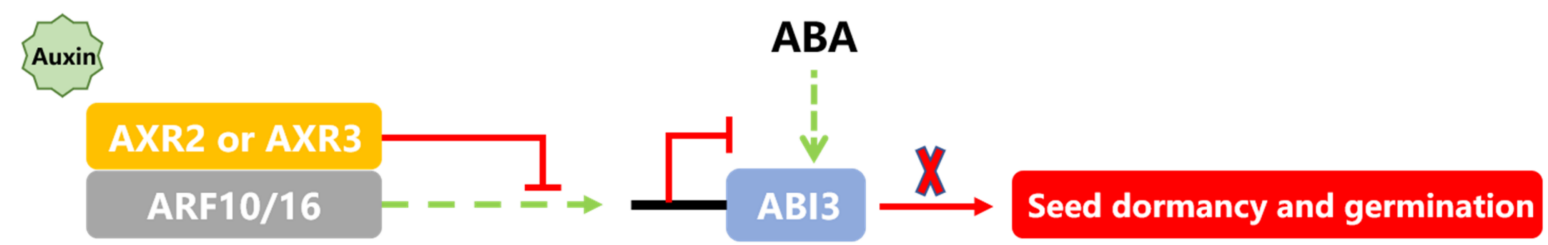

\section{Auxin signaling activation}

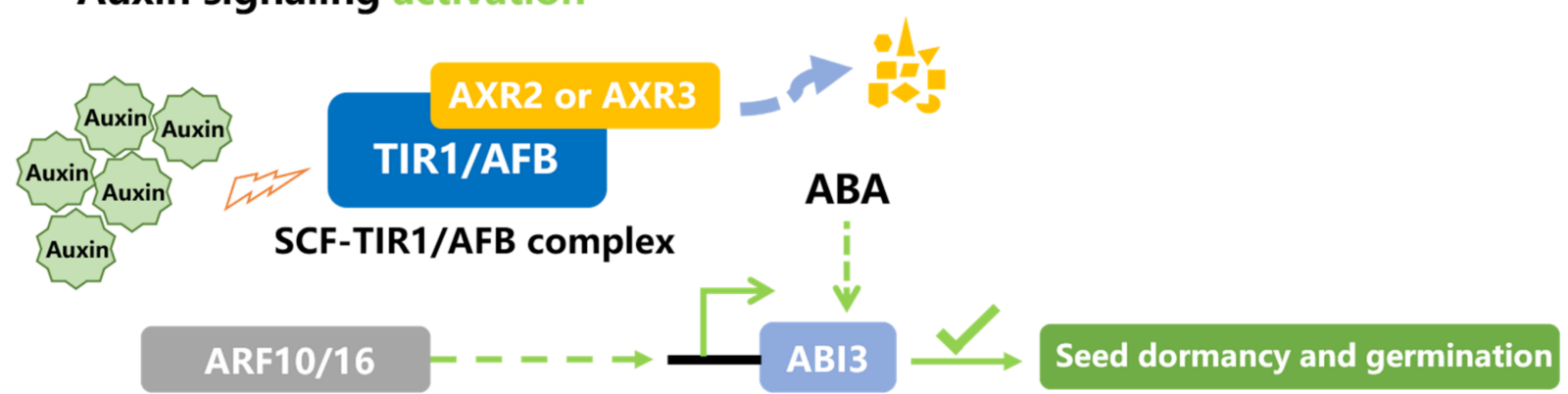

Figure 4. A proposed model for the effect of the ABA-auxin interaction on the control of seed dormancy/germination modified, modified on the basis of the work of Liu et al. [60]. The solid arrows and lines indicate direct regulation, and the dotted arrows indicate indirect regulation. The $\mathrm{ABI} 3$ box indicates the $\mathrm{ABI} 3$ gene region.

Some valuable research results can sometimes provide us with some inspiration and with the next research direction. For example, Lu et al. [62] found that the co-processing of $\mathrm{ABA}$ and IAA can affect the color and softening phenotype of bananas by regulating the expression of chlorophyll degradation-related genes, carotenoid biosynthesis-related genes, and pectin degradation-related genes. They revealed that ABA and IAA may regulate the postharvest ripening process of bananas through antagonistic interactions. Whether this 
effect is direct or indirect requires further exploration. The relevant genes or proteins may be directly regulated by hormonal molecules, while if they are indirectly regulated, ARFs are likely to be important participants. Studying the mechanism of ARFs at the molecular level will help us better understand the regulatory effect of auxin on plants.

\section{Challenges and Outlooks}

Currently, increasingly more studies have found evidence of ARF involvement in the regulation of plant growth and development, but the exploration of deeper molecular mechanisms and the construction of regulatory networks are not well developed. One of the major challenges for the future is to define the specific functions of each individual ARF gene during plant growth and development. The auxin signal transduction pathway mediated by ARFs is very complex, and we still need to further systematically study the target genes of ARFS in the auxin pathway, such as SAUR, GH3, and AUX/IAA, which will help us to have a more comprehensive and deeper understanding of the role of ARFS in plant growth, development, and environmental adaptation. As early as 2004, Tian et al. [63] suggested that in Arabidopsis ARF8 may control free IAA levels in a negative feedback manner by regulating GH3 gene expression, which may be important for maintaining auxin homeostasis. Advancing the study of negative feedback mechanisms will facilitate our comprehensive understanding of the auxin signal transduction pathway. In addition, further research on the deterministic interactions between transcription factors and transcription factors, as well as between transcription factors and genes, is needed. For example, some researchers found significant changes in the expression of ARF genes in banana and grape, respectively, during fruit development and ripening [64]. However, we are not aware of any studies related to the involvement of ARFs in the fruit development and ripening mechanisms of these two fruits. Overexpression, RNAi, and genome-editing methods such as CRISPR-Cas9 should be applied more widely to investigate the function and mechanism of ARFs. In addition, the function of ARFs in some species is still in the initial stage, and only a few relevant studies have performed identification and differential expression analysis of ARF genes, such as litchi [15]. The potential role of ARFs in these species needs to be further explored and verified. After the ARFs genes have been identified, further work should focus on identifying the protein partners that bind to them and the genes that they directly target to regulate.

It is generally accepted that ARF family proteins and Aux/IAA family proteins are involved in the auxin signal transduction pathways in a mutually binding manner. In reality, however, the exact interaction between the two that actually occurs in plants is not known, and the specific effects of this interaction are not well understood. Many studies have been able to demonstrate theoretical interactions between ARF transcription factors and Aux/IAA family proteins, or with other proteins, through methods such as yeast two-hybrid crosses, but there is often a lack of more robust evidence to confirm whether such interactions actually occur in plants. We speculate that the binding between ARF and Aux/IAA should be dynamic and not static, exhibiting different levels of interaction in different environments, or even only under certain conditions (e.g., auxin concentrations below a threshold). Considering that the interaction between Aux/IAA protein and ARFs is widespread, a more complete research plan should be to simultaneously analyze the effects of Aux/IAA proteins interacting with ARFs when studying the regulation of ARFs on plant growth and development.

In addition, numerous studies have shown that an ARF protein can interact with multiple Aux/IAAs and vice versa. $\mathrm{Hu}$ [44] and $\mathrm{Xu}$ [65] demonstrated the interaction between IAA9 and ARF7/ ARF5 by yeast two-hybrid system (Y2H) experiments, respectively. A study has shown that ARFs that act as activators (e.g., PeARF14, SlARF6A) have a higher affinity for Aux/IAAs interactions compared to ARFs that act as repressors (e.g., PeARF24, SIARF2A). The phenomenon seems to be conserved in some species (e.g., tomato, Arabidopsis, and rice), but it is unknown as to whether this is the case across the plant kingdom [66-68]. The above evidence suggests that the auxin signaling pathway is com- 
plex, and that this 'multiple binding' of ARF and Aux/IAA proteins offers the theoretical possibility of competition between different transcription factors of the same family during auxin signaling, which may be necessary to maintain the accuracy and stability of auxin signaling. In addition to the potential competition between different ARFs during their interaction with Aux/IAA proteins, there is also potential competition between them in the regulation of downstream genes. Most ARFs bind directly to the auxin response element (AuxRE) on the promoter of the responding genes to exert regulatory functions. In tomato, it has been shown that both SIARF6A and SIARF10 can directly target the promoter of SlGLK1, and that both SIARF6A and SIARF24 bind to the SIPIN1 promoter. However, these competitions have not been adequately studied and could be the focus of future research on the actual function of genes and proteins. Since multiple ARFs and Aux/IAAs may be involved in the regulation of a single life activity, their full identification would be a major effort. It is worth considering the development of an effective research system to address this issue.

The most important significance of scientific and technological progress is to improve human life. Solving the practical problems facing mankind should be the ultimate goal of all scientific work. We hope that the subsequent research on ARFs will be able to provide us with more consideration to the guiding value of fruit tree breeding, fruit and vegetable preservation, and engineering construction. For example, there has been much interest in improving seed germination, fruit yield and nutritional value, pest and disease resistance, and salt and drought tolerance in crops, and this will help us to improve breeding strategies. Of course, this may also require the use of gene editing and other tools to achieve our aims. Moreover, research methods need to be constantly innovated. In addition to the application of some traditional research methods such as $\mathrm{Y} 1 \mathrm{H}$ (yeast one-hybrid system), $\mathrm{Y} 2 \mathrm{H}$, bioinformatics analysis, and gene expression level determination, we are able to see that some new techniques such as CRISPR gene editing are also increasingly being used in the study of ARFs. Bouzroud et al. [52] successfully downregulated SIARF4 expression in tomato using CRISPR technology instead of antisense technology for gene manipulation. Using a transgenic approach, Liu et al. [32] investigated the fact that ARF6 and ARF8 have conserved roles in controlling growth and development of vegetative and flower organs in dicots through transgenic expression of Arabidopsis thaliana MIR167a in tomato. We look forward to more techniques being applied to the identification and functional study of ARFs.

Author Contributions: Conceptualization, X.K. and X.Z.; methodology, Z.X.; software, B.W.; investigation, C.W. (Chao Wang) and J.Z.; resources, C.W. (Caie Wu); data curation, S.Y.; writing-original draft preparation, X.Z.; writing - review and editing, X.K.; supervision, Z.X.; project administration, X.K.; funding acquisition, X.K. All authors have read and agreed to the published version of the manuscript.

Funding: Please add: This research was funded by National Natural Science Foundation of China, China, grant number 32072274 and 31871848.

Institutional Review Board Statement: Not applicable.

Informed Consent Statement: Not applicable.

Acknowledgments: Thanks to Zhang Xujiao for the language polish of this article.

Conflicts of Interest: The authors declare no conflict of interest.

\section{References}

1. Weijers, D.; Wagner, D. Transcriptional Responses to the Auxin Hormone. Annu. Rev. Plant Biol. 2016, 67, 539-574. [CrossRef] [PubMed]

2. Salehin, M.; Bagchi, R.; Estelle, M. SCFTIR1/AFB-Based Auxin Perception: Mechanism and Role in Plant Growth and Development. Plant Cell 2015, 27, 9-19. [CrossRef] [PubMed] 
3. Korasick, D.A.; Westfall, C.S.; Lee, S.G.; Nanao, M.H.; Dumas, R.; Hagen, G.; Guilfoyle, T.J.; Jez, J.M.; Strader, L.C. Molecular basis for AUXIN RESPONSE FACTOR protein interaction and the control of auxin response repression. Proc. Natl. Acad. Sci. USA 2014, 111, 5427-5432. [CrossRef] [PubMed]

4. Kato, H.; Mutte, S.K.; Suzuki, H.; Crespo, I.; Das, S.; Radoeva, T.; Fontana, M.; Yoshitake, Y.; Hainiwa, E.; van den Berg, W.; et al. Design principles of a minimal auxin response system. Nat Plants 2020, 6, 473. [CrossRef]

5. Estrada-Johnson, E.; Csukasi, F.; Pizarro, C.M.; Vallarino, J.G.; Kiryakova, Y.; Vioque, A.; Brumos, J.; Medina-Escobar, N.; Botella, M.A.; Alonso, J.M.; et al. Transcriptomic Analysis in Strawberry Fruits Reveals Active Auxin Biosynthesis and Signaling in the Ripe Receptacle. Front. Plant Sci. 2017, 8, 889. [CrossRef]

6. Guan, C.M.; Wu, B.B.; Yu, T.; Wang, Q.Q.; Krogan, N.T.; Liu, X.G.; Jiao, Y.L. Spatial Auxin Signaling Controls Leaf Flattening in Arabidopsis. Curr. Biol. 2017, 27, 2940-2950. [CrossRef]

7. Schuetz, M.; Fidanza, M.; Mattsson, J. Identification of Auxin Response Factor-Encoding Genes Expressed in Distinct Phases of Leaf Vein Development and with Overlapping Functions in Leaf Formation. Plants 2019, 8, 242. [CrossRef]

8. Wilmoth, J.C.; Wang, S.C.; Tiwari, S.B.; Joshi, A.D.; Hagen, G.; Guilfoyle, T.J.; Alonso, J.M.; Ecker, J.R.; Reed, J.W. NPH4/ARF7 and ARF19 promote leaf expansion and auxin-induced lateral root formation. Plant J. 2005, 43, 118-130. [CrossRef]

9. Wu, L.J.; Tian, Z.D.; Zhang, J.H. Functional Dissection of Auxin Response Factors in Regulating Tomato Leaf Shape Development Front. Plant Sci. 2018, 9, 957. [CrossRef]

10. Yuan, Y.J.; Xu, X.; Gong, Z.H.; Tang, Y.W.; Wu, M.B.; Yan, F.; Zhang, X.L.; Zhang, Q.; Yang, F.Q.; Hu, X.W.; et al. Auxin response factor 6A regulates photosynthesis, sugar accumulation, and fruit development in tomato. Hortic. Res. 2019, 6, 1-16. [CrossRef]

11. Zhang, H.H.; Li, L.L.; He, Y.Q.; Qin, Q.Q.; Chen, C.H.; Wei, Z.Y.; Tan, X.X.; Xie, K.L.; Zhang, R.F.; Hong, G.J.; et al. Distinct modes of manipulation of rice auxin response factor OsARF17 by different plant RNA viruses for infection. Proc. Natl. Acad. Sci. USA 2020, 117, 9112-9121. [CrossRef] [PubMed]

12. Ellis, C.M.; Nagpal, P.; Young, J.C.; Hagen, G.; Guilfoyle, T.J.; Reed, J.W. AUXIN RESPONSE FACTOR1 and AUXIN RESPONSE FACTOR2 regulate senescence and floral organ abscission in Arabidopsis thaliana. Development 2005, 132, 4563-4574. [CrossRef] [PubMed]

13. Wang, S.X.; Shi, F.Y.; Dong, X.X.; Li, Y.X.; Zhang, Z.H.; Li, H. Genome-wide identification and expression analysis of auxin response factor (ARF) gene family in strawberry (Fragaria vesca). J. Integr. Agr. 2019, 18, 1587-1603. [CrossRef]

14. Rena, Z.; Liu, R.; Gu, W.; Dong, X. The Solanum lycopersicum auxin response factor SlARF2 participates in regulating lateral root formation and flower organ senescence. Plant Sci. 2017, 256, 103-111. [CrossRef] [PubMed]

15. Zhang, Y.Q.; Zeng, Z.H.; Chen, C.J.; Li, C.Q.; Xia, R.; Li, J.G. Genome-wide characterization of the auxin response factor (ARF) gene family of litchi (Litchi chinensis Sonn.): Phylogenetic analysis, miRNA regulation and expression changes during fruit abscission. Peerj 2019, 7, e6677. [CrossRef] [PubMed]

16. Wang, J.W.; Wang, L.J.; Mao, Y.B.; Cai, W.J.; Xue, H.W.; Chen, X.Y. Control of root cap formation by microRNA-targeted auxin response factors in Arabidopsis. Plant Cell 2005, 17, 2204-2216. [CrossRef]

17. Waller, F.; Furuya, M.; Nick, P. OsARF1, an auxin response factor from rice, is auxin-regulated and classifies as a primary auxin responsive gene. Plant Mol. Biol. 2002, 50, 415-425. [CrossRef]

18. Qi, Y.H.; Wang, S.K.; Shen, C.J.; Zhang, S.N.; Chen, Y.; Xu, Y.X.; Liu, Y.; Wu, Y.R.; Jiang, D.A. OsARF12, a transcription activator on auxin response gene, regulates root elongation and affects iron accumulation in rice (Oryza sativa). New Phytol. 2012, 193, 109-120. [CrossRef]

19. Goh, T.; Kasahara, H.; Mimura, T.; Kamiya, Y.; Fukaki, H. Multiple AUX/IAA-ARF modules regulate lateral root formation: The role of Arabidopsis SHY2/IAA3-mediated auxin signalling. Philos. Trans. R. Soc. B Biol. Sci. 2012, 367, 1461-1468. [CrossRef]

20. Kalve, S.; Sizani, B.L.; Markakis, M.N.; Helsmoortel, C.; Vandeweyer, G.; Laukens, K.; Sommen, M.; Naulaerts, S.; Vissenberg, K.; Prinsen, E.; et al. Osmotic stress inhibits leaf growth of Arabidopsis thaliana by enhancing ARF-mediated auxin responses. New Phytol. 2020, 226, 1766-1780. [CrossRef]

21. Mallory, A.C.; Bartel, D.P.; Bartel, B. MicroRNA-directed regulation of Arabidopsis AUXIN RESPONSE FACTOR17 is essential for proper development and modulates expression of early auxin response genes. Plant Cell 2005, 17, 1360-1375. [CrossRef] [PubMed]

22. Inukai, Y.; Sakamoto, T.; Ueguchi-Tanaka, M.; Shibata, Y.; Gomi, K.; Umemura, I.; Hasegawa, Y.; Ashikari, M.; Kitano, H.; Matsuoka, M. Crown rootless1, which is essential for crown root formation in rice, is a target of an AUXIN RESPONSE FACTOR in auxin signaling. Plant Cell 2005, 17, 1387-1396. [CrossRef] [PubMed]

23. Yang, C.X.; Xu, M.; Xuan, L.; Jiang, X.M.; Huang, M.R. Identification and expression analysis of twenty ARF genes in Populus. Gene 2014, 544, 134-144. [CrossRef] [PubMed]

24. Herud, O.; Weijers, D.; Lau, S.; Jurgens, G. Auxin responsiveness of the MONOPTEROS-BODENLOS module in primary root initiation critically depends on the nuclear import kinetics of the Aux/IAA inhibitor BODENLOS. Plant J. 2016, 85, $269-277$. [CrossRef]

25. Dastidar, M.G.; Scarpa, A.; Magele, I.; Ruiz-Duarte, P.; von Born, P.; Bald, L.; Jouannet, V.; Maizel, A. ARF5/MONOPTEROS directly regulates miR390 expression in the Arabidopsis thaliana primary root meristem. Plant Direct 2019, 3, e00116. [CrossRef]

26. Schlereth, A.; Moller, B.; Liu, W.L.; Kientz, M.; Flipse, J.; Rademacher, E.H.; Schmid, M.; Jurgens, G.; Weijers, D. MONOPTEROS controls embryonic root initiation by regulating a mobile transcription factor. Nature 2010, 464, 913-916. [CrossRef] 
27. Zhang, K.; Wang, R.Z.; Zi, H.L.; Li, Y.P.; Cao, X.W.; Li, D.M.; Guo, L.; Tong, J.H.; Pan, Y.Y.; Jiao, Y.L.; et al. AUXIN RESPONSE FACTOR3 Regulates Floral Meristem Determinacy by Repressing Cytokinin Biosynthesis and Signaling. Plant Cell 2018, 30, 324-346. [CrossRef]

28. Yamaguchi, N.; Wu, M.F.; Winter, C.M.; Berns, M.C.; Nole-Wilson, S.; Yamaguchi, A.; Coupland, G.; Krizek, B.A.; Wagner, D. A Molecular Framework for Auxin-Mediated Initiation of Flower Primordia. Dev. Cell 2013, 24, 271-282. [CrossRef]

29. Nagpal, P.; Ellis, C.M.; Weber, H.; Ploense, S.E.; Barkawi, L.S.; Guilfoyle, T.J.; Hagen, G.; Alonso, J.M.; Cohen, J.D.; Farmer, E.; et al. Auxin response factors ARF6 and ARF8 promote jasmonic acid production and flower maturation. Development 2005, 132, 4107-4118. [CrossRef]

30. Ghelli, R.; Brunetti, P.; Napoli, N.; De Paolis, A.; Cecchetti, V.; Tsuge, T.; Serino, G.; Matsui, M.; Mele, G.; Rinaldi, G.; et al. A Newly Identified Flower-Specific Splice Variant of AUXIN RESPONSE FACTOR8 Regulates Stamen Elongation and Endothecium Lignification in Arabidopsis. Plant Cell 2018, 30, 620-637. [CrossRef]

31. Yang, J.; Tian, L.; Sun, M.X.; Huang, X.Y.; Zhu, J.; Guan, Y.F.; Jia, Q.S.; Yang, Z.N. AUXIN RESPONSE FACTOR17 Is Essential for Pollen Wall Pattern Formation in Arabidopsis. Plant Physiol 2013, 162, 720-731. [CrossRef] [PubMed]

32. Liu, N.; Wu, S.; Van Houten, J.; Wang, Y.; Ding, B.; Fei, Z.J.; Clarke, T.H.; Reed, J.W.; van der Knaap, E. Down-regulation of AUXIN RESPONSE FACTORS 6 and 8 by microRNA 167 leads to floral development defects and female sterility in tomato. J. Exp. Bot. 2014, 65, 2507-2520. [CrossRef] [PubMed]

33. Kumar, R.; Tyagi, A.K.; Sharma, A.K. Genome-wide analysis of auxin response factor (ARF) gene family from tomato and analysis of their role in flower and fruit development. Mol. Genet. Genom. 2011, 285, 245-260. [CrossRef] [PubMed]

34. Sun, R.R.; Wang, K.B.; Guo, T.L.; Jones, D.C.; Cobb, J.; Zhang, B.H.; Wang, Q.L. Genome-wide identification of auxin response factor (ARF) genes and its tissue-specific prominent expression in Gossypium raimondii. Funct. Integr. Genom. 2015, 15, 481-493. [CrossRef]

35. Pattison, R.J.; Csukasi, F.; Catala, C. Mechanisms regulating auxin action during fruit development. Physiol. Plant. 2014, 151, 62-72. [CrossRef]

36. Breitel, D.A.; Chappell-Maor, L.; Meir, S.; Panizel, I.; Puig, C.P.; Hao, Y.W.; Yifhar, T.; Yasuor, H.; Zouine, M.; Bouzayen, M.; et al. AUXIN RESPONSE FACTOR 2 Intersects Hormonal Signals in the Regulation of Tomato Fruit Ripening. PLoS Genet. 2016, 12, e1005903. [CrossRef]

37. Hao, Y.W.; Hu, G.J.; Breitel, D.; Liu, M.C.; Mila, I.; Frasse, P.; Fu, Y.Y.; Aharoni, A.; Bouzayen, M.; Zouine, M. Auxin Response Factor SlARF2 Is an Essential Component of the Regulatory Mechanism Controlling Fruit Ripening in Tomato. PLoS Genet. 2015, 11, e1005649. [CrossRef]

38. Waters, M.T.; Moylan, E.C.; Langdale, J.A. GLK transcription factors regulate chloroplast development in a cell-autonomous manner. Plant J. 2008, 56, 432-444. [CrossRef]

39. Sagar, M.; Chervin, C.; Roustan, J.P. Under-expression of the Auxin Response Factor Sl-ARF4 improves postharvest behavior of tomato fruits. Plant Signal. Behav. 2013, 8, e25647. [CrossRef]

40. Yuan, Y.J.; Mei, L.H.; Wu, M.B.; Wei, W.; Shan, W.; Gong, Z.H.; Zhang, Q.; Yang, F.Q.; Yan, F.; Zhang, Q.; et al. SIARF10, an auxin response factor, is involved in chlorophyll and sugar accumulation during tomato fruit development. J. Exp. Bot. 2018, 69, 5507-5518.

41. Hendelman, A.; Buxdorf, K.; Stav, R.; Kravchik, M.; Arazi, T. Inhibition of lamina outgrowth following Solanum lycopersicum AUXIN RESPONSE FACTOR 10 (SlARF10) derepression. Plant Mol. Biol. 2012, 78, 561-576. [CrossRef] [PubMed]

42. Liu, S.Y.; Zhang, Y.W.; Feng, Q.S.; Qin, L.; Pan, C.T.; Lamin-Samu, A.T.; Lu, G. Tomato AUXIN RESPONSE FACTOR 5 regulates fruit set and development via the mediation of auxin and gibberellin signaling. Sci. Rep. 2018, 8, 2971. [CrossRef] [PubMed]

43. De Jong, M.; Wolters-Arts, M.; Feron, R.; Mariani, C.; Vriezen, W.H. The Solanum lycopersicum auxin response factor 7 (SlARF7) regulates auxin signaling during tomato fruit set and development. Plant J. 2009, 57, 160-170. [CrossRef] [PubMed]

44. Hu, J.H.; Israeli, A.; Ori, N.; Sun, T.P. The Interaction between DELLA and ARF/IAA Mediates Crosstalk between Gibberellin and Auxin Signaling to Control Fruit Initiation in Tomato. Plant Cell 2018, 30, 1710-1728. [CrossRef]

45. De Jong, M.; Wolters-Arts, M.; Schimmel, B.C.J.; Stultiens, C.L.M.; de Groot, P.F.M.; Powers, S.J.; Tikunov, Y.M.; Bovy, A.G.; Mariani, C.; Vriezen, W.H.; et al. Solanum lycopersicum AUXIN RESPONSE FACTOR 9 regulates cell division activity during early tomato fruit development. J. Exp. Bot. 2015, 66, 3405-3416. [CrossRef]

46. Goetz, M.; Vivian-Smith, A.; Johnson, S.D.; Koltunow, A.M. AUXIN RESPONSE FACTOR8 is a negative regulator of fruit initiation in Arabidopsis. Plant Cell 2006, 18, 1873-1886. [CrossRef]

47. Li, S.B.; OuYang, W.Z.; Hou, X.J.; Xie, L.L.; Hu, C.G.; Zhang, J.Z. Genome-wide identification, isolation and expression analysis of auxin response factor (ARF) gene family in sweet orange (Citrus sinensis). Front. Plant Sci. 2015, 6, 119. [CrossRef]

48. Kuang, J.F.; Wu, J.Y.; Zhong, H.Y.; Li, C.Q.; Chen, J.Y.; Lu, W.J.; Li, J.G. Carbohydrate Stress Affecting Fruitlet Abscission and Expression of Genes Related to Auxin Signal Transduction Pathway in Litchi. Int. J. Mol. Sci. 2012, 13, 16084-16103. [CrossRef]

49. He, Q.G.; Hong, K.Q.; Zou, R.; Liao, F.; Cui, S.F.; Zhang, E.Z.; Huang, M.K. The role of jasmonic acid and lipoxygenase in propylene-induced chilling tolerance on banana fruit. Eur. Food Res. Technol. 2014, 238, 71-78. [CrossRef]

50. Wang, Y.C.; Wang, N.; Xu, H.F.; Jiang, S.H.; Fang, H.C.; Su, M.Y.; Zhang, Z.Y.; Zhang, T.L.; Chen, X.S. Auxin regulates anthocyanin biosynthesis through the Aux/IAA-ARF signaling pathway in apple. Hortic. Res. 2018, 5, 1-11. [CrossRef] 
51. Narise, T.; Kobayashi, K.; Baba, S.; Shimojima, M.; Masuda, S.; Fukaki, H.; Ohta, H. Involvement of auxin signaling mediated by IAA14 and ARF7/19 in membrane lipid remodeling during phosphate starvation. Plant Mol. Biol. 2010, 72, 533-544. [CrossRef] [PubMed]

52. Bouzroud, S.; Gasparini, K.; Hu, G.J.; Barbosa, M.A.M.; Rosa, B.L.; Fahr, M.; Bendaou, N.; Bouzayen, M.; Zsogon, A.; Smouni, A.; et al. Down Regulation and Loss of Auxin Response Factor 4 Function Using CRISPR/Cas9 Alters Plant Growth, Stomatal Function and Improves Tomato Tolerance to Salinity and Osmotic Stress. Genes 2020, 11, 272. [CrossRef] [PubMed]

53. Bouzroud, S.; Gouiaa, S.; Hu, N.; Bernadac, A.; Mila, I.; Bendaou, N.; Smouni, A.; Bouzayen, M.; Zouine, M. Auxin Response Factors (ARFs) are potential mediators of auxin action in tomato response to biotic and abiotic stress (Solanum lycopersicum). PLoS ONE 2018, 13, e0193517. [CrossRef] [PubMed]

54. Jain, M.; Khurana, J.P. Transcript profiling reveals diverse roles of auxin-responsive genes during reproductive development and abiotic stress in rice. FEBS J. 2009, 276, 3148-3162. [CrossRef]

55. Du, H.; Liu, H.B.; Xiong, L.Z. Endogenous auxin and jasmonic acid levels are differentially modulated by abiotic stresses in rice. Front. Plant Sci. 2013, 4, 397. [CrossRef] [PubMed]

56. Chen, X.; Liu, Z.P.; Shi, G.Q.; Bai, Q.Q.; Guo, C.J.; Xiao, K. MIR167a transcriptionally regulates ARF6 and ARF8 and mediates drastically plant Pi-starvation response via modulation of various biological processes. Plant Cell Tissue Organ Cult. 2018, 133, 177-191. [CrossRef]

57. Kang, C.; He, S.Z.; Zhai, H.; Li, R.J.; Zhao, N.; Liu, Q.C. A Sweetpotato Auxin Response Factor Gene (IbARF5) Is Involved in Carotenoid Biosynthesis and Salt and Drought Tolerance in Transgenic Arabidopsis. Front. Plant Sci. 2018, 9, 1307. [CrossRef]

58. Li, J.S.; Dai, X.H.; Zhao, Y.D. A role for auxin response factor 19 in auxin and ethylene signaling in Arabidopsis. Plant Physiol. 2006, 140, 899-908. [CrossRef]

59. Wang, L.; Hua, D.P.; He, J.N.; Duan, Y.; Chen, Z.Z.; Hong, X.H.; Gong, Z.Z. Auxin Response Factor2 (ARF2) and Its Regulated Homeodomain Gene HB33 Mediate Abscisic Acid Response in Arabidopsis. PLoS Genet. 2011, 7, e1002172. [CrossRef]

60. Liu, X.D.; Zhang, H.; Zhao, Y.; Feng, Z.Y.; Li, Q.; Yang, H.Q.; Luan, S.; Li, J.M.; He, Z.H. Auxin controls seed dormancy through stimulation of abscisic acid signaling by inducing ARF-mediated ABI3 activation in Arabidopsis. Proc. Natl. Acad. Sci. USA 2013, 110, 15485-15490. [CrossRef]

61. Yue, P.T.; Lu, Q.; Liu, Z.; Lv, T.X.; Li, X.Y.; Bu, H.D.; Liu, W.T.; Xu, Y.X.; Yuan, H.; Wang, A.D. Auxin-activated MdARF5 induces the expression of ethylene biosynthetic genes to initiate apple fruit ripening. New Phytol. 2020, 226, 1781-1795. [CrossRef] [PubMed]

62. Lu, W.J.; Mao, L.C.; Chen, J.X.; Han, X.Y.; Ren, X.C.; Ying, T.J.; Luo, Z.S. Interaction of abscisic acid and auxin on gene expression involved in banana ripening. Acta Physiol. Plant 2018, 40, 1-9. [CrossRef]

63. Tian, C.; Muto, H.; Higuchi, K.; Matamura, T.; Tatematsu, K.; Koshiba, T.; Yamamoto, K.T. Disruption and overexpression of auxin response factor 8 gene of Arabidopsis affect hypocotyl elongation and root growth habit, indicating its possible involvement in auxin homeostasis in light condition. Plant J. 2004, 40, 333-343. [CrossRef] [PubMed]

64. Wan, S.B.; Li, W.L.; Zhu, Y.Y.; Liu, Z.M.; Huang, W.D.; Zhan, J.C. Genome-wide identification, characterization and expression analysis of the auxin response factor gene family in Vitis vinifera. Plant Cell Rep. 2014, 33, 1365-1375. [CrossRef] [PubMed]

65. Xu, C.Z.; Shen, Y.; He, F.; Fu, X.K.; Yu, H.; Lu, W.X.; Li, Y.L.; Li, C.F.; Fan, D.; Wang, H.C.; et al. Auxin-mediated Aux/IAA-ARF-HB signaling cascade regulates secondary xylem development in Populus. New Phytol. 2019, 222, 752-767. [CrossRef]

66. Piya, S.; Shrestha, S.K.; Binder, B.; Stewart, C.N.; Hewezi, T. Protein-protein interaction and gene co-expression maps of ARFs and Aux/IAAs in Arabidopsis. Front. Plant Sci. 2014, 5, 744. [CrossRef]

67. Kumar, R.; Agarwal, P.; Pareek, A.; Tyagi, A.K.; Sharma, A.K. Genomic Survey, Gene Expression, and Interaction Analysis Suggest Diverse Roles of ARF and Aux/IAA Proteins in Solanaceae. Plant Mol. Biol. Rep. 2015, 33, 1552-1572. [CrossRef]

68. Shen, C.J.; Wang, S.K.; Bai, Y.H.; Wu, Y.R.; Zhang, S.N.; Chen, M.; Guilfoyle, T.J.; Wu, P.; Qi, Y.H. Functional analysis of the structural domain of ARF proteins in rice (Oryza sativa L.). J. Exp. Bot. 2010, 61, 3971-3981. [CrossRef] 